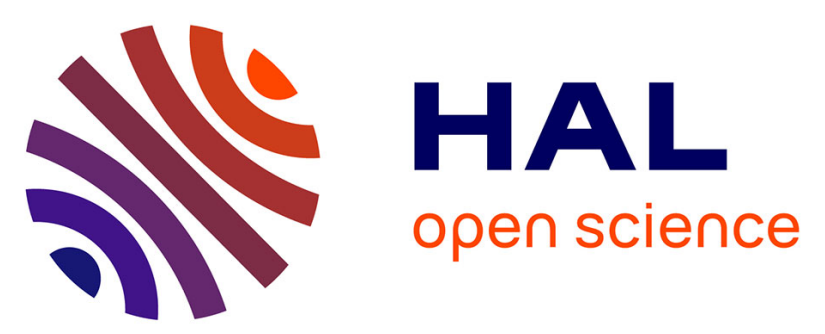

\title{
Effets d'un bioadjuvant sur les caractéristiques de surface de pâtes cimentaires
}

Charlotte Munzer, Essia Belhaj, Thierry T. Meylheuc, André Lecomte, Françoise Feugeas

\section{To cite this version:}

Charlotte Munzer, Essia Belhaj, Thierry T. Meylheuc, André Lecomte, Françoise Feugeas. Effets d'un bioadjuvant sur les caractéristiques de surface de pâtes cimentaires. Matériaux \& Techniques, 2015, 103 (2), 10.1051/mattech/2015024 . hal-02637138

\section{HAL Id: hal-02637138 \\ https://hal.inrae.fr/hal-02637138}

Submitted on 27 May 2020

HAL is a multi-disciplinary open access archive for the deposit and dissemination of scientific research documents, whether they are published or not. The documents may come from teaching and research institutions in France or abroad, or from public or private research centers.
L'archive ouverte pluridisciplinaire HAL, est destinée au dépôt et à la diffusion de documents scientifiques de niveau recherche, publiés ou non, émanant des établissements d'enseignement et de recherche français ou étrangers, des laboratoires publics ou privés.

$$
\text { Copyright }
$$




\title{
Effets d'un bioadjuvant sur les caractéristiques de surface de pâtes cimentaires
}

\author{
C. Munzer ${ }^{1}$, E. Belhaj ${ }^{1,2}$, T. Meylheuc ${ }^{3}$, A. Lecomte $^{4}$ et F. Feugeas ${ }^{1}$
}

Reçu le 12 février 2015, accepté le 23 avril 2015

\begin{abstract}
Résumé - L'objectif de cet article est d'étudier l'influence d'un bioadjuvant (BA) sur les caractéristiques de surface des pâtes cimentaires. Les caractéristiques de surface des pâtes de ciment bioadjuvantées sont étudiées avec la mesure de l'angle de contact dynamique. Ces essais ont été réalisés pour comparer le comportement de pâtes cimentaires sèches avec 1,5\% de BA et sans bioadjuvant lorsqu'une goutte d'eau est déposée sur leur surface. Les résultats montrent que le bioadjuvant diminue les vitesses d'évolution des angles de contact. Une technique originale d'analyse de la pénétration par rapport à l'étalement s'affranchissant du paramètre temps a été développée. Elle a permis de mettre en évidence que le bioadjuvant favorise l'étalement de la goutte et diminue le phénomène de pénétration au sein du matériau.
\end{abstract}

Mots clés : Bioadjuvant / bioréceptivité / pâte de ciment / surface / angle de contact

\begin{abstract}
Effects of bioadmixture on surface characteristics of cement pastes. The aim of this paper is to study the effect of a bioadmixture (BA) on the surface characteristics of cement pastes. Surfaces of bioadmixtured cement pastes are studied by the measurement of the dynamic contact angle. These tests were performed to compare the behavior of dry cement pastes with $1.5 \%$ and without bioadmixture when a drop of water is deposited on the sample surface. The results show that the bioadmixture reduces the evolution speed of the contact angles. An original technique for analyzing the penetration compared to spreading has been developed while avoiding time constraint. It highlighted that bioadmixture promotes spreading of the drop and reduces the phenomenon of penetration into the material.
\end{abstract}

Key words: Bioadmixture / bioreceptivity / cement paste / surface / contact angle

\section{Introduction}

Le béton est le matériau le plus utilisé dans le monde. Son impact environnemental, de la fabrication du ciment jusqu'à la réalisation de l'ouvrage et lors de sa durée de vie, est capital.

Le béton est sensible à différentes attaques qui peuvent altérer sa qualité et sa durée de vie. Il existe différents types de mécanismes d'altération, les altérations physiques et les altérations chimiques. Les altérations chimiques peuvent être d'origine interne (alcali-réaction) ou externe (carbonatation). Parmi les différentes agressions que le béton peut subir au cours du temps, la biodétérioration peut accélérer la dégradation des ouvrages. Ce phénomène est lié à la croissance de micro-organismes sur la surface des bétons et mortiers [1] qui, par leur présence, modifient les échanges entre le matériau et l'environnement. Comme tous les matériaux présents dans un environnement naturel, les bétons, mortiers et pâtes de ciments présentent une bioréceptivité : aptitude d'un matériau à être colonisé par des organismes vivants $[2,3]$. Ce phénomène dépend de l'état de surface, notamment de la rugosité du matériau, de son $\mathrm{pH}$ et de sa porosité [4] mais également des types de micro-organismes [5] présents dans le milieu considéré [6]. Les conséquences de la bio-colonisation d'une surface de béton vont d'une simple altération esthétique à une dégradation des parements et à un risque pour l'ouvrage.

\footnotetext{
${ }^{1}$ ICube UMR 7357, INSA de Strasbourg, CNRS, 24 boulevard de la Victoire, 67084 Strasbourg Cedex, France charlotte.munzer@insa-strasbourg.fr

2 IRISE, EICESI Strasbourg, 2 allée des Foulons, 67831 Lingolsheim, France

3 INRA UMR 1319, INRA Paristech, MICALIS, Equipe B2HM, 25 avenue de la République, 91300 Massy, France

4 Nancy Université UMR 7198 IJL-CP2S-207, IUT de Nancy-Brabois dpt GC, Le Montet, Rue du Doyen Urion, 54600 Villers-les-Nancy, France
} 
Tableau 1. Composition chimique du CEM I ( $\%$ massique).

Table 1. Chemical composition of CEM I (mass \%).

\begin{tabular}{ccccccccccccc}
\hline $\mathrm{SiO}_{2}$ & $\mathrm{Al}_{2} \mathrm{O}_{3}$ & $\mathrm{Fe}_{2} \mathrm{O}_{3}$ & $\mathrm{CaO}$ & $\mathrm{MgO}$ & $\mathrm{SO}_{3}$ & $\mathrm{~K}_{2} \mathrm{O}$ & $\mathrm{Na}_{2} \mathrm{O}$ & $\mathrm{S}^{-}$ & $\mathrm{Cl}^{-}$ & $\mathrm{CO}_{2}$ & $\mathrm{CaO}^{\text {libre }}$ & $\mathrm{Na}_{2} \mathrm{O}^{\text {éq actif }}$ \\
\hline 20,3 & 5,1 & 3,4 & 63,3 & 1,5 & 3,5 & 1 & 0,14 & 0 & 0,05 & 0,6 & 0,9 & 0,79 \\
\hline
\end{tabular}

De plus, la présence de biofilms peut induire un risque sanitaire, les ouvrages de rétention ou d'adduction d'eau étant souvent réalisés en béton.

C'est pourquoi il apparaît utile de développer des bétons dont les surfaces peuvent présenter des caractéristiques d'inhibition de contamination biologique.

Un matériau bio-sourcé a été mis au point dans le but d'être incorporé aux formules de bétons afin de leur conférer de telles propriétés. Il est fabriqué à partir de produits issus de la matrice extracellulaire de certaines bactéries. L'utilisation de ce type de produit comme adjuvant dans les bétons a fait l'objet d'études précédentes $[7,8]$. Le produit biosourcé étudié a été préalablement testé pour vérifier la possibilité de l'utiliser en tant qu'adjuvant pour les bétons au sens de la norme [NF EN 480] [9]. Les biofilms se développent sur des surfaces grâce à l'adhésion des micro-organismes. Les étapes de formation des biofilms font l'objet de nombreuses recherches [10-13]. Parmi les paramètres influençant la bioadhésion se trouvent les propriétés physico-chimiques de la surface colonisée, notamment sa rugosité, mais également sa composition chimique, son caractère plus ou moins hydrophile. .

La technique consistant à déposer une goutte d'eau sur la surface étudiée peut alors permettre d'obtenir diverses informations importantes utiles à la compréhension de sa bioréceptivité.

Hamadi et al. [14] ont notamment corrélé les valeurs d'angle de contact de surfaces colonisées par différentes espèces bactériennes avec leur caractère hydrophobe. Absolom et al. [15] ont analysé les relations entre l'adhésion bactérienne et l'énergie de surface de différents couples (solution bactérienne et support), corrélées à la mesure de l'angle de contact. Al-Radha et al. [16] ont, quant à eux, étudié des surfaces de différents matériaux céramiques (à base de zirconium et de titanium) et ont déterminé que de plus faibles énergies de surface et mouillabilités du matériau engendraient une plus faible adhésion bactérienne.

L'objectif de cette étude est ainsi d'évaluer l'effet du bioadjuvant sur l'évolution des angles de contact d'une goutte d'eau sur des pâtes cimentaires. Les mesures d'angles dynamiques ont été réalisées sur les pâtes cimentaires contenant 0 et 1,5\% de bioadjuvant. L'évolution des angles et des diamètres de la goutte a été observée au cours du temps. Ces deux informations ont permis d'évaluer et de différencier les phénomènes d'étalement et de pénétration de la goutte. Une technique originale d'analyse de la pénétration par rapport à l'étalement s'affranchissant du paramètre temps a été développée. Elle a permis de mettre en évidence que le bioadjuvant favorise l'étalement de la goutte et diminue le phénomène de pénétration au sein du matériau.

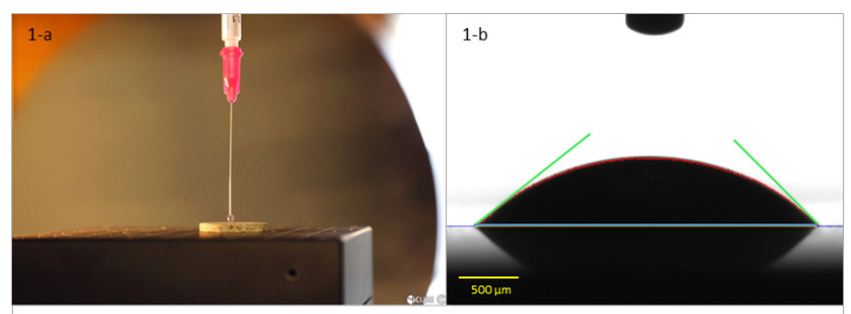

$1-\mathrm{c}$

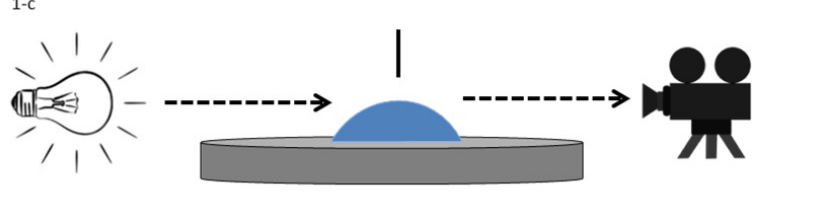

Fig. 1. (a) goutte déposée à la surface de l'échantillon de pâte de ciment; (b) profil de goutte sur un échantillon; (c) principe de mesure.

Fig. 1. (a) drop on cement paste sample; (b) drop profile; (c) measurement device.

\section{Matériaux et méthodes}

\subsection{Matériaux}

Les pâtes de ciment ont été réalisées en respectant la norme [NF EN 196-3]. Le ciment utilisé est du CEM I 52,5R CE CP2 dont la caractérisation chimique est indiquée dans le tableau 1.

Pour le gâchage des pâtes de ciment, l'eau distillée $(\mathrm{pH}=5,8)$ a été utilisée avec un rapport $E / C=$ 0,315 déterminé grâce à l'essai de consistance Vicat [NF EN 196].

Le bioadjuvant est un produit issu des substances extracellulaires des bactéries qui se présente sous forme liquide. Il est incorporé dans la masse au moment du gâchage en tant qu'adjuvant au sens de la norme [NF EN 934-2] c'est-à-dire à des dosages inférieurs à $5 \%$ de la masse de ciment. Les dosages sont de $0 \%$ et de $1,5 \%$.

\subsection{Techniques expérimentales}

\subsubsection{Mesure de l'angle de contact dynamique}

Une goutte d'eau de volume $V$ (entre 2 et $4 \mu \mathrm{L}$ ) est déposée sur la surface de l'échantillon à $t=0$.

Un analyseur de profil de goutte DSA30 Kruss permet, grâce à une caméra (12 images par seconde), d'observer l'évolution du profil d'une goutte d'eau déposée à la surface de l'échantillon poreux (Fig. 1).

Une vidéo est enregistrée et le traitement de celle-ci (logiciel DSA 4) permet d'évaluer les angles de contact et le diamètre de la goutte. 


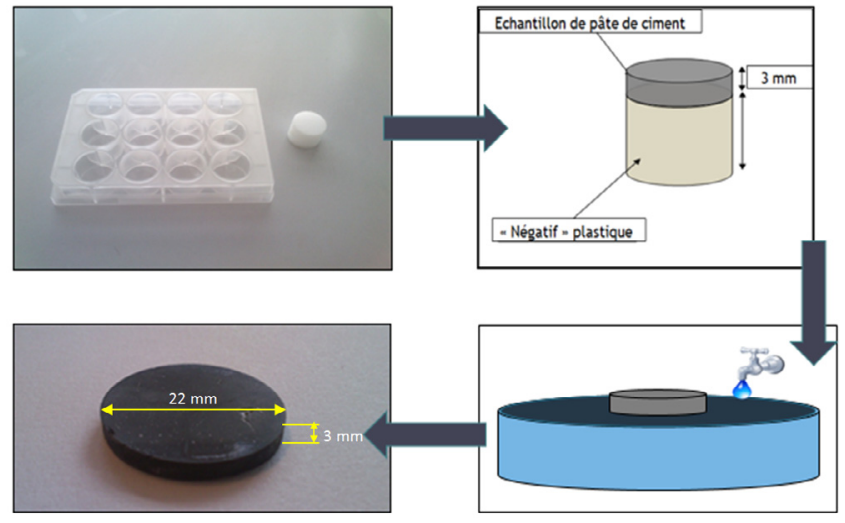

Fig. 2. Dispositif de réalisation des pastilles de pâte de ciment. Fig. 2. Cement paste samples moulding device.

\subsubsection{Protocole de réalisation et de conservation des échantillons}

Les échantillons de forme cylindrique présentent une épaisseur maximale de $3 \mathrm{~mm}$ et un diamètre de $22 \mathrm{~mm}$. Ces caractéristiques géométriques sont optimales pour permettre d'étudier ultérieurement la bioréceptivité des échantillons. Un protocole de réalisation des échantillons détaillé dans un précédent article [17] est résumé sur la figure 2. Immédiatement après le mélange eau + ciment + BA, la pâte de ciment est coulée dans les moules : une plaque multipuits pour culture cellulaire, permettant de réaliser 12 pastilles simultanément. L'épaisseur inférieure à $3 \mathrm{~mm}$ est obtenue grâce à un négatif réalisé en polyoxyméthylène. Puis les échantillons sont stockés en salle de conservation $\left(T=20{ }^{\circ} \mathrm{C} \pm 2^{\circ}\right.$ et humidité relative $>80 \%$ ) durant $24 \mathrm{~h}$; les pastilles sont ensuite démoulées et stockées dans de l'eau distillée filtrée à $0,22 \mu \mathrm{m}$, avec un rapport eau/solide en masse de 1 pour 1 . Après 3 jours, les échantillons sont polis sous eau avec 3 papiers abrasifs PRESI de grains successifs (p320, p600 et p1200 P) jusqu'à obtention d'une surface plane et lisse. Ils sont ensuite passés aux ultrasons 10 min pour éliminer les résidus du polissage et stockés à nouveau dans de l'eau distillée filtrée.

Ce protocole permet de garantir un état de surface similaire entre tous les échantillons.

Préalablement à la mesure, les échantillons sont repolis au papier abrasif (PRESI) de grain le plus fin (1200 p) et passés aux ultrasons pour éliminer les éventuels dépôts et cristallisations en surface. Les échantillons, dont le $\mathrm{pH}$ de surface est supérieur à 12 , sont alors placés en étuve ventilée à $50{ }^{\circ} \mathrm{C}$ jusqu'à stabilisation de leur masse ( $\Delta<0,01 \%$ de la masse initiale de l'échantillon saturé) entre 2 pesées successives espacées d'une heure (adaptation de la norme [NF P EN 18-459] pour la détermination de la teneur en eau des granulats).

Une fois sortis de l'étuve, les échantillons secs sont placés en enceinte pour la mesure des angles de contact, la température ambiante et l'humidité relative sont vérifiées.

Les mesures ont été réalisées après 58 jours de cure humide. Les essais ont été réalisés à une température de

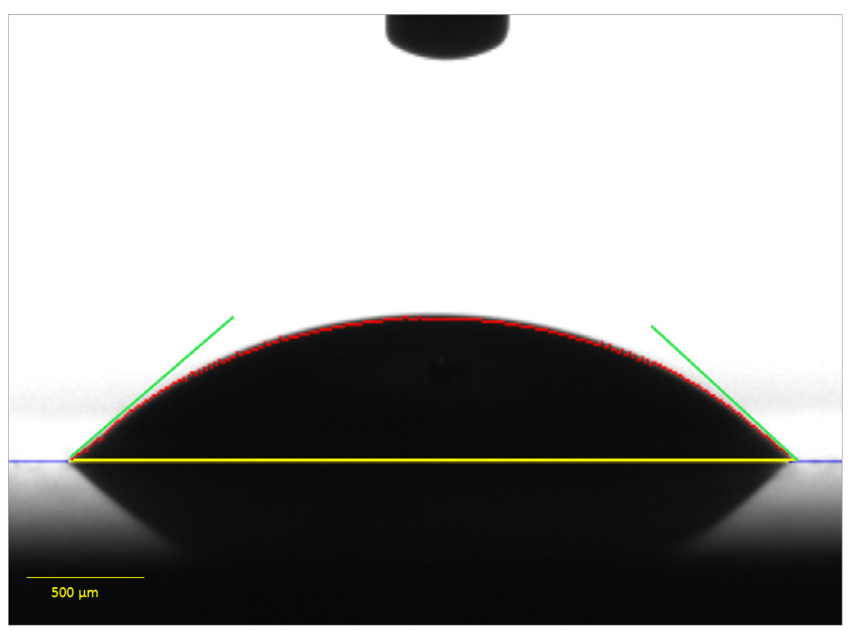

Fig. 3. Profil de goutte.

Fig. 3. Drop profile.

$27{ }^{\circ} \mathrm{C} \pm 1^{\circ}$ et à une humidité relative de $64 \% \pm 2 \%$. Six échantillons de chaque dosage ont été testés, avec 3 ou 4 gouttes par échantillon afin d'assurer une bonne répétabilité des résultats.

\section{Résultats et discussion}

\section{1 Évolution des angles de contact en fonction du temps}

Le profil d'une goutte d'eau sur une pastille est représenté dans la figure 3 qui correspond à une capture d'image à $t=28 \mathrm{~s}$ après la dépose de la goutte.

Le matériau étudié présentant une surface rugueuse et hétérogène, on constate, sur certaines valeurs mesurées à des instants donnés, une dissymétrie entre les angles gauches et droits. Une telle dissymétrie a également été mise en évidence avec cette technique par d'autres travaux $[18,19]$. Néanmoins, les droites représentant l'évolution de ces angles en fonction du temps montrent globalement des valeurs d'angles $(\theta \mathrm{g}$ et $\theta \mathrm{d})$ similaires puisqu'elles se superposent (Fig. 5).

La mesure d'angle de contact est couramment utilisée sur des surfaces lisses et non poreuses. Dans ce cas, le modèle de Young Dupré permet de déterminer l'énergie de la surface.

L'équation de Young Dupré, équation (1), définit la relation entre les 3 énergies d'équilibre du système LiquideSolide-Gaz et l'angle de contact statique. Les paramètres influençant la forme de la goutte sont les caractéristiques de la surface, le liquide utilisé et les conditions extérieures (Fig. 4).

$$
\gamma_{\mathrm{LV}} \cos \theta=\gamma_{\mathrm{SV}}-\gamma_{\mathrm{SL}}
$$

$\theta$ est l'angle de contact entre la goutte et la surface, $\gamma_{\mathrm{LV}}$ est la tension superficielle Liquide/Vapeur, $\gamma_{\mathrm{SL}}$ est la tension superficielle Solide/Liquide et $\gamma_{\mathrm{SV}}$ est la tension superficielle Solide/Vapeur. Elles s'expriment en N/m. 


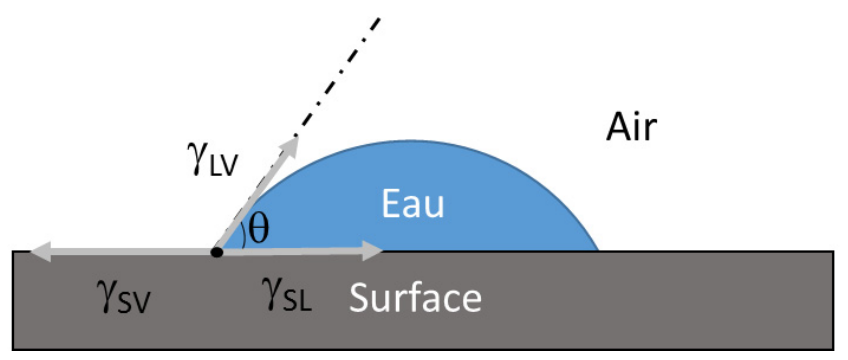

Fig. 4. Profil d'une goutte d'eau sur une surface, modèle de Young-Dupré.

Fig. 4. Water drop profile on surface, Young-Dupré model.

Il existe d'autre modèles qui prennent respectivement en compte l'hétérogénéité chimique et la porosité de surface comme le modèle de Cassie ou Cassie-Baxter, mais ils ne sont pas valables lorsque le liquide pénètre au sein de l'échantillon testé [20]. Le modèle de Wenzel intègre la rugosité de la surface par le biais d'un facteur de rugosité dépendant de la résolution avec laquelle il est mesuré [21]. Pour ces raisons, de tels modèles n'ont pas été utilisés pour tenter de déterminer l'énergie de surface d'un matériau aussi complexe, seule l'évolution des angles de contact est étudiée.

Les valeurs initiales des angles de contact varient entre $37^{\circ}$ et $66^{\circ}$ pour les pastilles sans BA et entre $35^{\circ}$ et $62^{\circ}$ pour les pastilles avec 1,5\% de BA. Ces valeurs sont approximatives puisque les angles sont dynamiques et varient très rapidement dans les premières secondes. Pour s'affranchir de l'effet dynamique, la technique de la bulle captive est souvent utilisée. Dans ce cas, l'échantillon est immergé dans l'eau distillée afin de saturer les pores et éviter ainsi la pénétration. Une bulle d'air est insufflée sous l'échantillon via une aiguille courbe et l'angle de contact statique est mesuré. Ces essais sont en cours et ne font pas l'objet de cette étude. De plus, après $100 \mathrm{~s}$ généralement, les angles atteignent des valeurs très faibles et ne peuvent être mesurés par l'appareil. Il n'y a donc pas de différence remarquable entre les valeurs initiales des angles de contact en fonction du taux de BA.

La figure 5 présente un exemple de l'évolution des angles de contact (droite et gauche) en fonction du temps pour un échantillon à $0 \%$ et un échantillon à $1,5 \%$ de BA.

Les résultats montrent que les angles de contact suivent une évolution linéaire au cours du temps. La diminution des angles de contact pour l'échantillon sans bioadjuvant apparaît plus rapide que celle pour l'échantillon avec 1,5\% de bioadjuvant.

Afin de déterminer les vitesses de diminution des angles de contact, le coefficient directeur de la droite représentative de l'évolution de celui-ci au cours de temps est déterminé. Étant donné l'hétérogénéité de surface des échantillons, de nombreuses mesures ont été effectuées et seules les pentes calculées présentant un coefficient de détermination $R^{2}<0,9$ ont été gardées. Ce tri a permis de conserver 21 mesures pour les échantillons à $0 \%$ et 25 pour les échantillons avec 1,5\% de BA. Les moyennes des pentes sont représentées sur la figure 6. Elle montre que la présence du bioadjuvant modifie de manière significative la vitesse de diminution des angles de contact : avec 1,5\% de bioadjuvant, les angles diminuent 2,5 fois moins vite que sans bioadjuvant.

Cette diminution des angles correspond à une combinaison de l'étalement de la goutte sur la surface de la pastille et de sa pénétration dans la pâte cimentaire poreuse (Fig. 7).

\section{2 Évolution des angles en fonction du diamètre}

Une technique originale d'analyse de la pénétration par rapport à l'étalement s'affranchissant du paramètre temps a été développée, il s'agit ici de distinguer les deux comportements résultant de (1) la pénétration de la goutte au sein de l'échantillon et (2) l'étalement de la goutte à la surface de l'échantillon (Fig. 8).

En supposant que la goutte pénètre (Fig. 8a), $\theta$, l'angle de contact, diminue quand $D$, le diamètre de contact, diminue. Au contraire, si la goutte ne fait que s'étaler (Fig. 8b) $\theta$ diminue quand $D$ augmente. En réalité, c'est une combinaison des deux phénomènes qui a lieu lorsqu'une goutte d'eau est déposée sur la surface des échantillons de pâte cimentaire (Fig. 8c).

Plusieurs auteurs ont déjà utilisé l'hypothèse simplificatrice d'une goutte représentée par une calotte sphérique $[22,23]$. L'évolution théorique des angles en fonction du diamètre peut être alors déterminée pour la pénétration grâce à l'équation (2) (Fig. 9).

$$
d=D \sin \theta
$$

Pour l'étalement de la goutte conservant son volume, il peut alors être assimilé au volume d'une calotte sphérique figure 10, équation (3).

$$
R=\sqrt[3]{\frac{6 V \text { calotte }}{\pi(1-\cos \theta)\left[3(\sin \theta)^{2}+(1-\cos \theta)^{2}\right]}}
$$

En remplaçant $D$ par $2 R$ dans l'équation (2), on obtient la relation entre $\theta$ et $d(=2 a)$.

Or $D=2 R$ et $d=2 a$ (Fig. 10), en combinant (3) et (2), on obtient l'équation (4), qui décrit la relation entre le rayon de contact a et l'angle de contact $\theta$.

$$
a=R \sin \theta .
$$

Cette équation permet de tracer l'angle de contact théorique en fonction du diamètre de contact théorique dans le cas de l'étalement pur.

Ces 2 courbes sont tracées sur la figure 11 avec un diamètre de départ arbitrairement fixé à $1 \mathrm{~mm}$.

Sur la figure 11, les deux comportements de la goutte explicités plus haut sont représentés. D'une part la pénétration pure, où on considère que l'angle et le diamètre diminuent ensemble, jusqu'à atteindre un diamètre et un angle de contact nul, et d'autre part l'étalement pur, où l'angle de contact va diminuer alors 
C. Munzer et al. : Matériaux \& Techniques 103, 208 (2015)

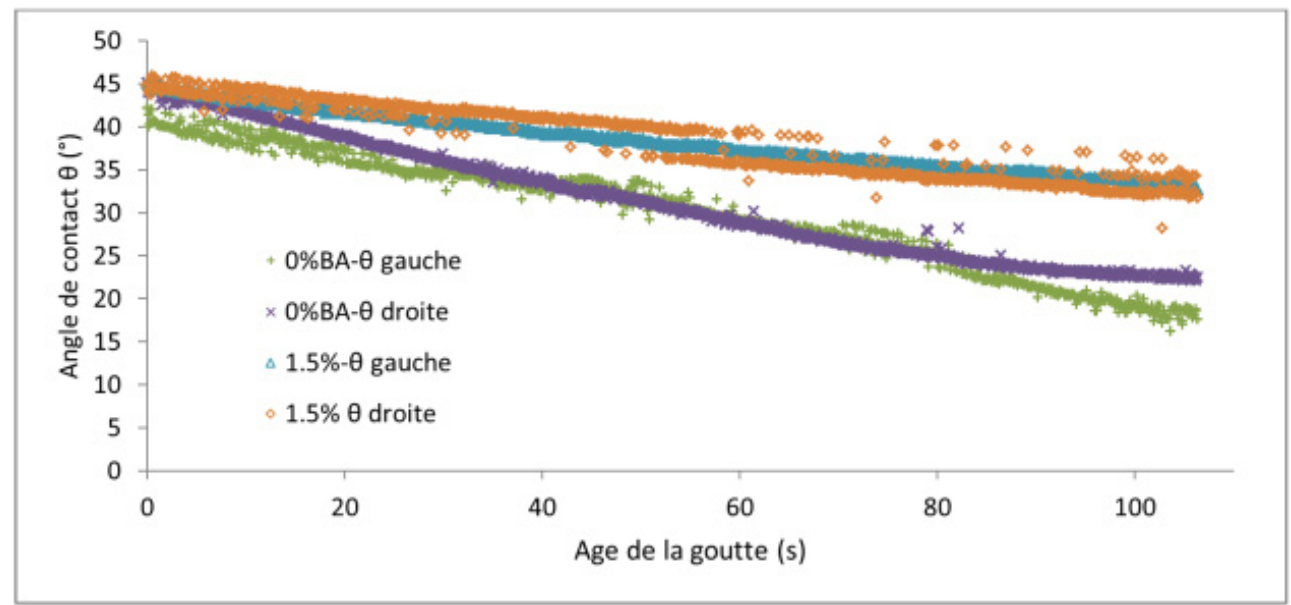

Fig. 5. Évolution des angles de contact en fonction du temps pour des pâtes sans BA et avec 1,5\% de BA Fig. 5. Evolution of contact angles vs. time for cement pastes without BA and with $1.5 \%$ of BA.

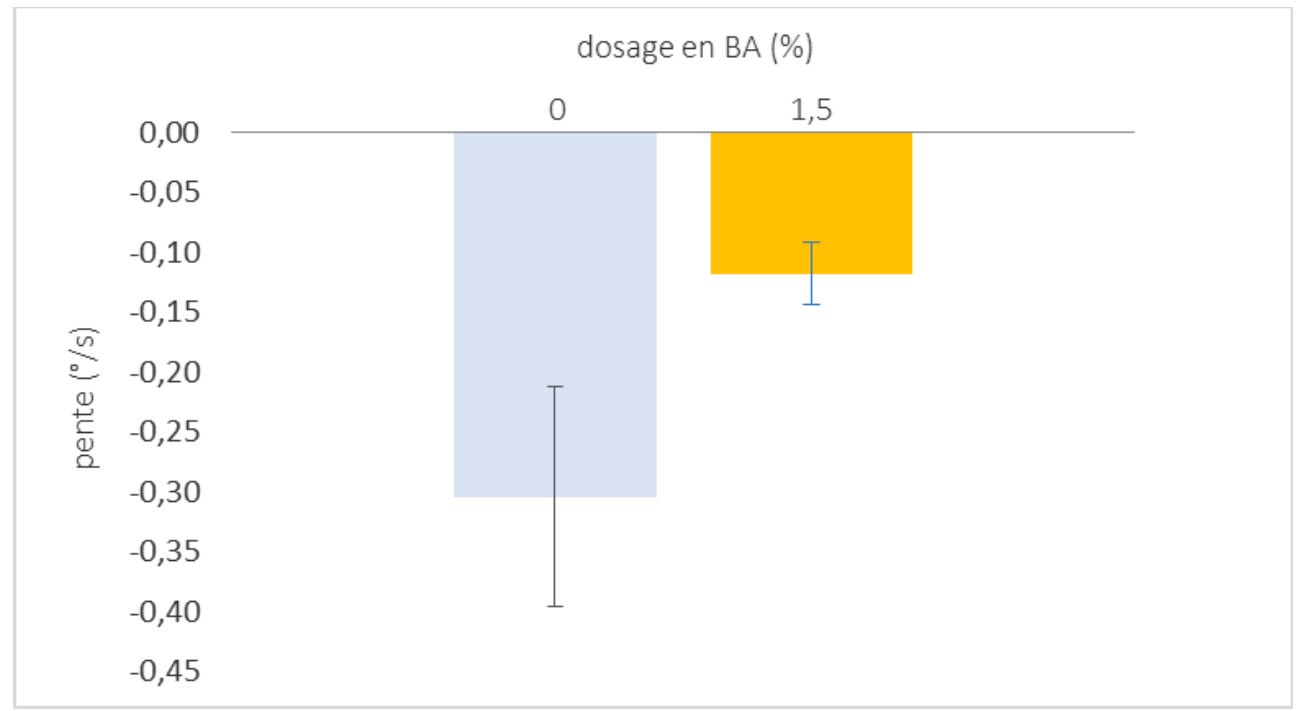

Fig. 6. Pentes moyennes représentant la vitesse de diminution des angles de contact d'une goutte d'eau sur une surface de pâte cimentaire.

Fig. 6. Average slopes representing the decreasing speed of contact angles of a water drop on a cementitious paste surface.
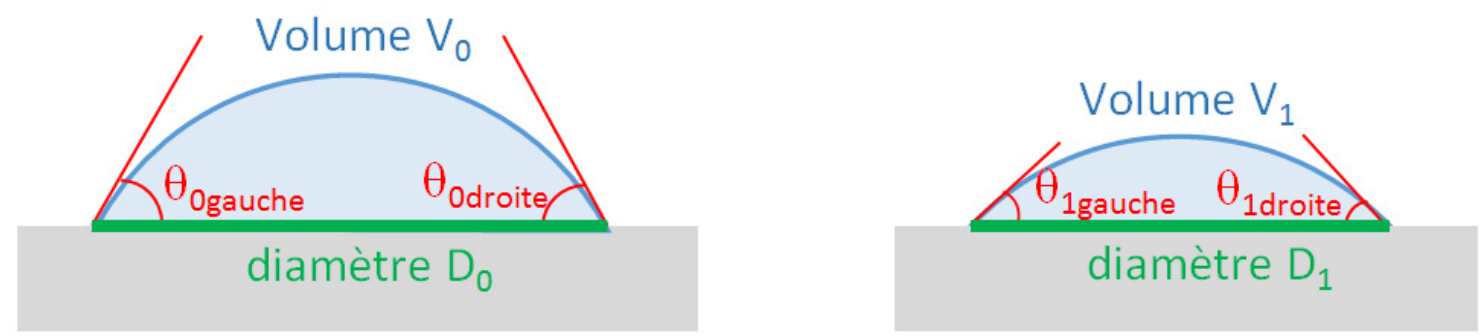

Fig. 7. Évolution du profil de la goutte au cours du temps.

Fig. 7. Evolution of the drop profile vs. time. 

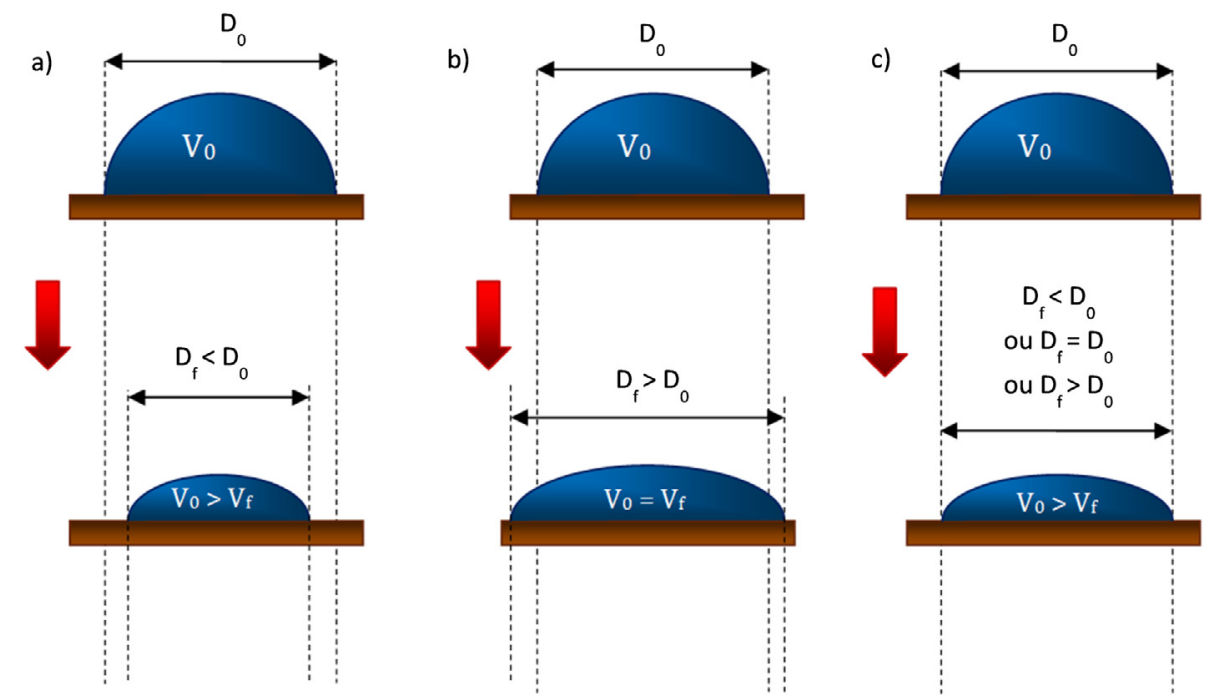

Fig. 8. Comportement théorique de la goutte déposée sur la pastille : (a) cas de la pénétration (b) cas de l'étalement, (c) combinaison étalement-pénétration.

Fig. 8. Theoritical behaviour of the drop on the sample surface: (a) in case of penetration, (b) in case of spreading, (c) spreadingpenetration combination.

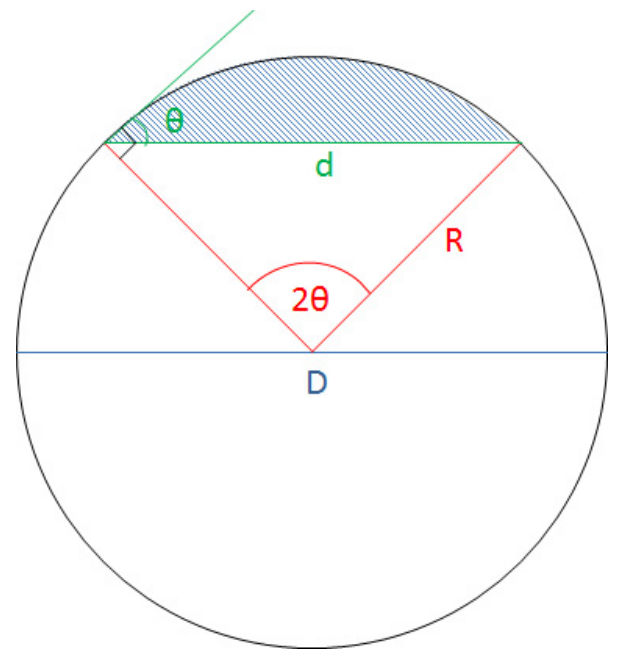

Fig. 9. Évolution de l'angle de contact en fonction du diamètre (pénétration).

Fig. 9. Evolution of contact angle vs. diameter (penetration).

que le diamètre de contact va augmenter jusqu'à atteindre un diamètre théoriquement infini.

Ce modèle analytique a été utilisé avec des résultats de la littérature. Kumar et al. [22] ont étudié le comportement de deux liquides (solution eau-alcool-polyvinyl : PVA et huile de silicone : SO) sur deux types de surface : une plaque de verre (non poreuse) et sur une couche unidirectionnelle de fibre de verre : UDMAT (poreuse). Sur la plaque de verre, une goutte de $\mathrm{SO}(V=5,9 \mu \mathrm{L})$ et une goutte de PVA $(V=7,8 \mu \mathrm{L})$ ont été déposées. Les données expérimentales de cet article ont été relevées afin de tracer l'évolution de l'angle de contact en fonction du diamètre en figure 12 , superposée avec la courbe

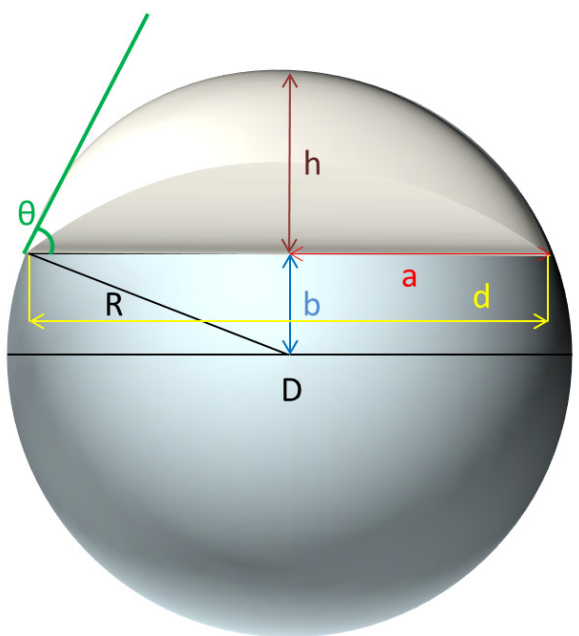

Fig. 10. Évolution du rayon $R$ de la sphère en fonction de l'angle de contact (étalement).

Fig. 10. Evolution of sphere radius $R$ vs. contact angle (spreading).

théorique démarrant aux valeurs d'angle et de diamètre expérimentales initiales.

L'évolution de l'angle de contact suit l'allure de la courbe théorique du modèle «étalement », la plaque de verre n'étant pas poreuse. Malgré l'hypothèse simplificatrice d'approximation de la goutte à une demi-sphère, le modèle théorique décrit donc bien la réalité de l'évolution de la goutte déposée sur une surface non poreuse.

Si la validation de la courbe théorique pour l'étalement est facile à obtenir au regard des résultats expérimentaux assez nombreux dans la littérature, il n'en est pas du tout de même pour la pénétration. Il n'existe pas de matériau n'offrant que de la pénétration à l'eau dans la 


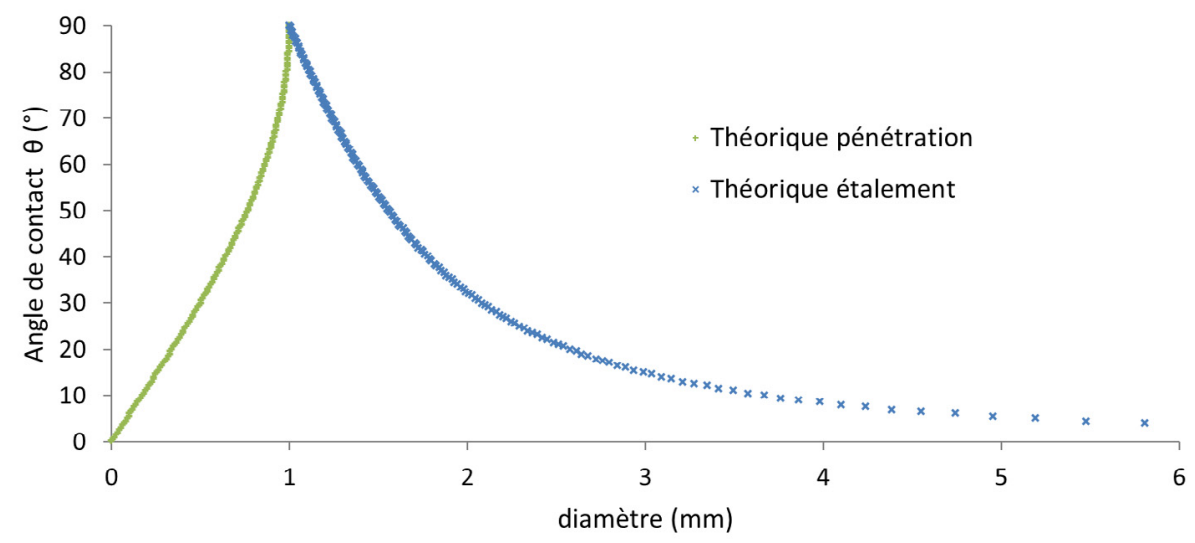

Fig. 11. Comportement théorique de la goutte déposée sur la surface de l'échantillon.

Fig. 11. Theoritical behaviour of the drop on the sample surface.

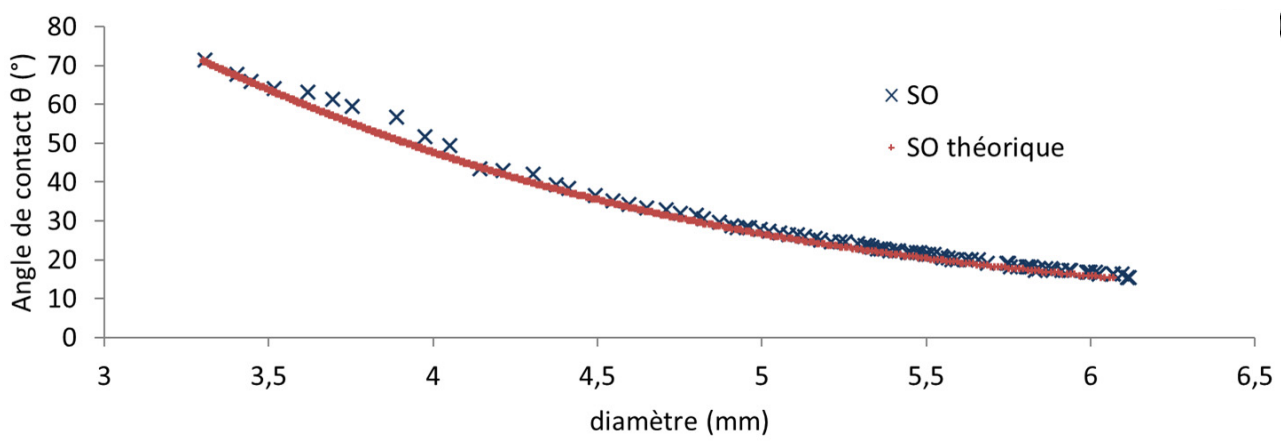

(a)

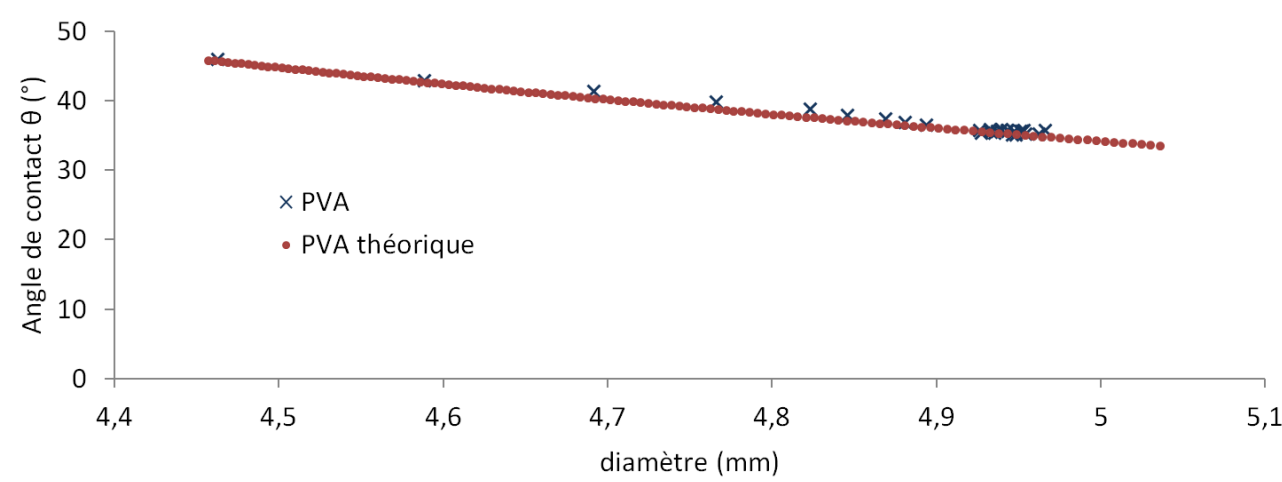

(b)

Fig. 12. (a) SO sur plaque de verre; (b) PVA sur plaque de verre.

Fig. 12. (a) SO on glass plate; (b) PVA on glass plate.

bibliographie qui a été effectuée. L'intérêt ici n'est pas de valider ce modèle mais de l'exploiter pour analyser plus finement le comportement dynamique d'une goutte en s'affranchissant du paramètre temps.

L'évolution des angles de contact en fonction du diamètre pour un échantillon à $0 \%$ de $\mathrm{BA}$ et un échantillon à 1,5\% de BA est présentée figure 13 .

Les deux essais ont duré 106 s. Pour l'échantillon sans BA, les valeurs des angles de contact se situent entre $49^{\circ}$ et $19^{\circ}$, ce qui correspond à une variation de $30^{\circ}$ et une évolution du diamètre de $0,4 \mathrm{~mm}$. Pour l'échantillon avec 1,5\% de BA, la différence des valeurs des angles n'est que de $10^{\circ}\left(36^{\circ}-26^{\circ}\right)$ et les valeurs de diamètres varient entre 3,44 et $3,48 \mathrm{~mm}$, ce qui correspond à une évolution de 0,04 mm. L'intervalle d'évolution des angles et du diamètre de l'échantillon avec 1,5\% BA est très petit par rapport à celui sans BA (3 fois moins pour les angles et 10 fois moins pour le diamètre) pour le même nombre de points mesurés. La figure 13 représente volontairement les deux courbes avec les mêmes échelles pour illustrer les différences importantes d'évolution des angles en fonction des diamètres. 


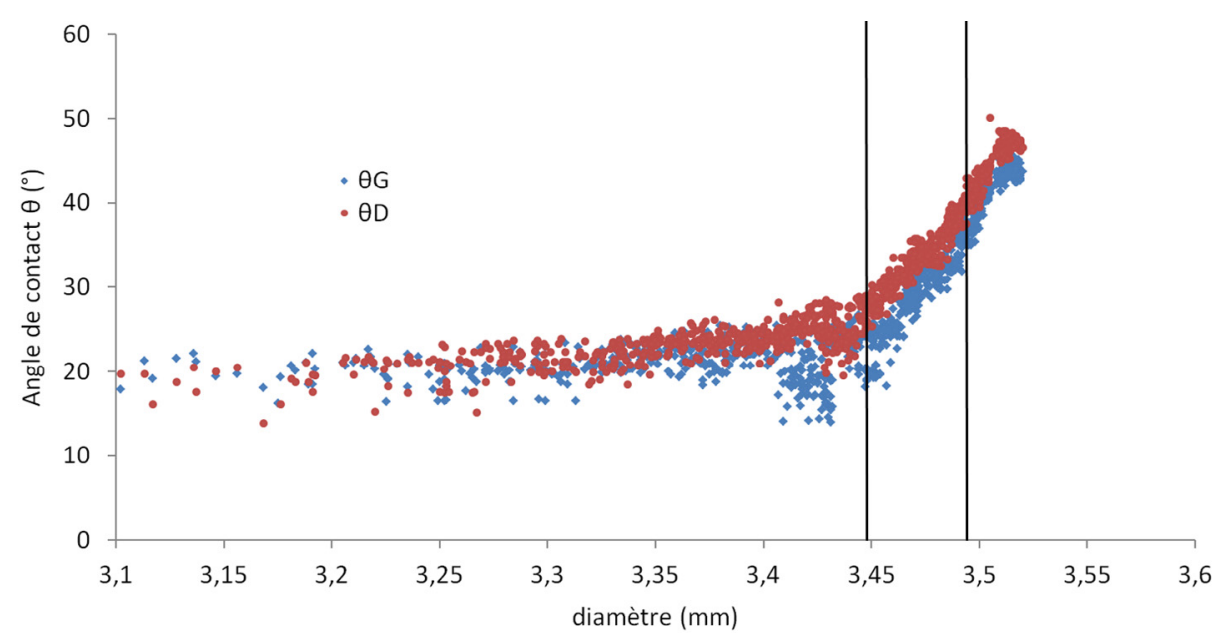

(a)

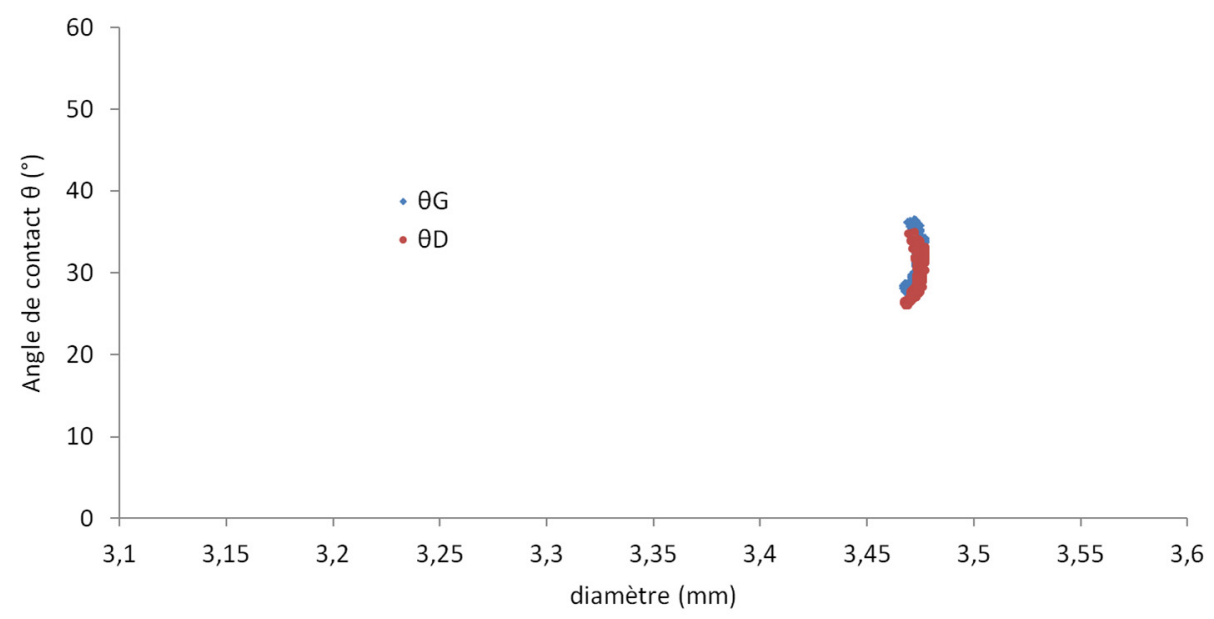

(b)

Fig. 13. (a) Comportement de la goutte sur échantillon à $0 \%$ de BA (a) et (b) sur un échantillon avec 1,5\% de BA. Fig. 13. (a) Drop behaviour on sample with $0 \%$ of $B A$ and (b) on sample with $1.5 \%$ of $B A$.

Pour la courbe de l'échantillon sans BA, 3 phases distinctes peuvent être observées : La première phase montre une diminution de l'angle rapide pour une faible diminution du diamètre, ce qui implique que la pénétration est prépondérante, l'étalement influence tout de même l'allure de la courbe $\theta=f(d)$. La deuxième phase présente un comportement semblable, c'est-à-dire que la diminution de l'angle est plus faible par rapport à la diminution du diamètre. Une moins forte influence de l'étalement que dans la première phase est visible, en effet la pente de cette partie linéaire diminue, se rapprochant plus du modèle théorique simplifié de pénétration pure. La troisième phase montre une diminution du diamètre avec un angle quasiment constant, ce qui signifie que le comportement de la goutte correspond presque uniquement à de la pénétration et que l'étalement n'a presque plus d'influence sur ce comportement. Trois phases ont également été mises en évidence grâce à l'analyse des courbes des diamètres en fonction du temps de liquides sur une surface poreuse $[20,21]$.
Pour pouvoir mieux observer les phases d'évolution des angles avec 1,5\% de BA, la figure $13 \mathrm{~b}$ a été mise à une échelle plus adaptée. La courbe est représentée sur la figure 14 .

Bien que 3 phases soient également observables, le comportement est différent : en effet, le diamètre commence ici par augmenter avec la diminution de l'angle, puis se stabilise alors que l'angle continue à diminuer pour finir par diminuer très légèrement avec l'angle. La présence de bioadjuvant favorise l'étalement de l'eau sur la surface, cet effet est particulièrement important dans la première phase correspondant aux angles les plus importants où l'étalement, c'est-à-dire l'augmentation du diamètre par rapport à l'angle, est tel que la pente de la courbe $\theta=f(D)$ change de signe par rapport à celle de l'échantillon sans BA (Fig. 13a) favorisant l'effet de la pénétration de la goutte.

Ces deux types de comportement s'observent pour 10 essais de chaque composition ( $0 \%$ et $1,5 \%$ ). De plus, les résultats de vitesse de diminution des angles de contact 


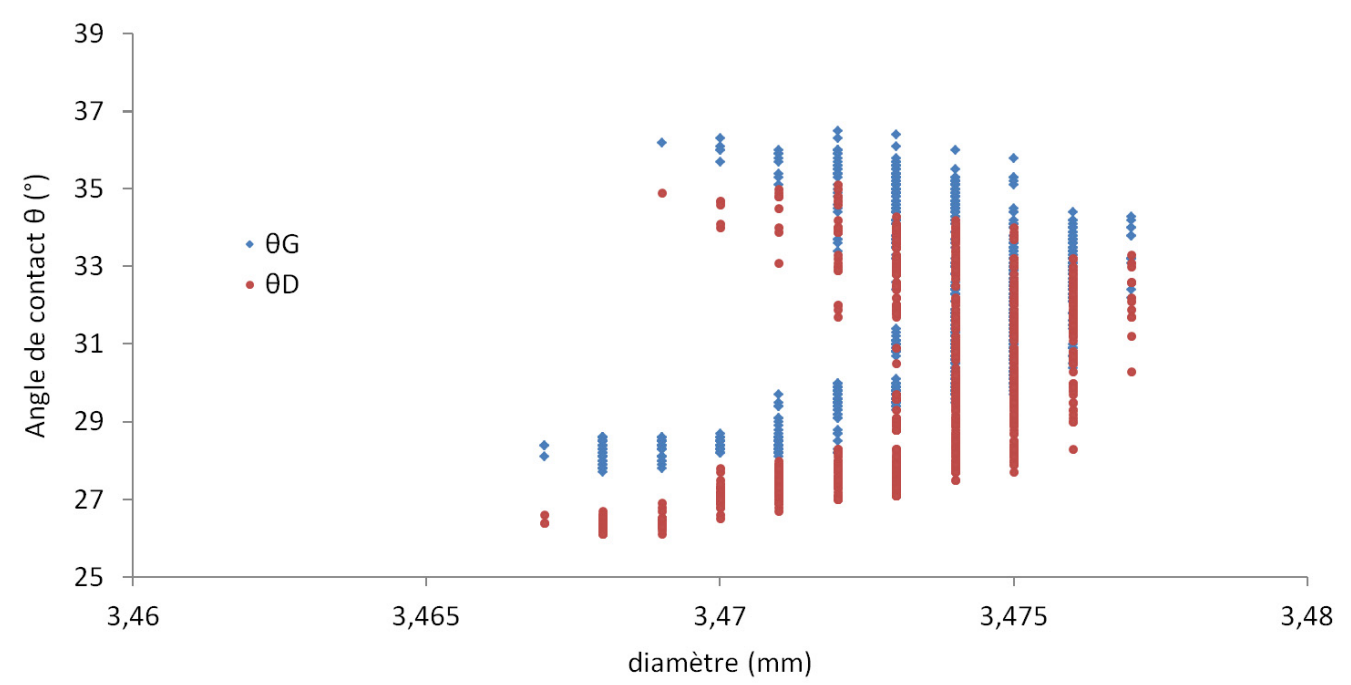

Fig. 14. Comportement de la goutte sur un échantillon avec 1,5\% de BA.

Fig. 14. Drop behaviour on sample with $1.5 \%$ of $B A$.

(Fig. 6) mettent en évidence un retard de vitesse de diminution en présence de BA ce qui tend à prouver que l'ajout de BA induit une diminution de la pénétration de la goutte d'eau liée plus à un phénomène de colmatage des porosités qu'à un effet d'hydrophobicité du produit. En effet, l'étalement de la goutte montre que la surface est mouillable.

Le modèle analytique défini plus haut a été utilisé avec des données extraites de courbes angles et diamètres en fonction du temps pour des surfaces poreuses testées par différentes équipes de recherche [20,21]. Kumar et al. [22] ont étudié 2 liquides (PVA et SO) sur une surface poreuse (UDMAT). Clarke et al. [23] ont utilisé une solution aqueuse de glycerol et d'hexylene glycol : G/HG sur du papier filtre présentant différentes tailles de porosités $(0,1 ; 0,22 ; 0,45$ et $0,65 \mu \mathrm{m})$. Les volumes des gouttes déposées sont de l'ordre de $100 \mathrm{pL}$.

On retrouve 3 phases comme observé sur la figure 14. Dans la première phase, l'étalement est prépondérant puisque le diamètre augmente avec la diminution de l'angle. La deuxième phase voit la pénétration devenir plus importante avec une quasi stabilisation du diamètre alors que l'angle continue de diminuer. Enfin lors de la troisième phase la pénétration domine l'étalement avec une diminution du diamètre plus forte et une diminution des angles plus faible voire nulle.

Sur la figure 15a, la goutte de PVA s'étale plus que la goutte de SO, phénomène qui s'explique par le caractère moins mouillant du SO. Sur la figure $15 \mathrm{~b}$, le comportement de la goutte de $\mathrm{G} / \mathrm{HG}$ sur le papier présentant la porosité la plus fine $(0,1 \mu \mathrm{m})$ semble s'orienter plus vers l'étalement que pour des porosités plus grossières.

Ces observations confortent les conclusions concernant l'effet du BA sur la porosité de la pâte cimentaire : une modification très légère de porosité peut favoriser l'étalement d'une goutte au profit de la pénétration, il faut évidemment tenir compte des effets d'échelles et de taille respective de la goutte par rapport au coefficient de rugosité par exemple.

\section{Conclusions}

- La présence de bioadjuvant dans la composition des pâtes de ciment modifie significativement leur comportement, plus particulièrement celui de leur surface en contact avec de l'eau. L'eau pénètre moins rapidement au sein des échantillons avec 1,5\% de BA.

- L'étude de l'évolution de l'angle de contact en fonction du diamètre a permis de distinguer les comportements d'étalement et de pénétration des gouttes d'eau. Un modèle analytique a été développé, il a été utilisé pour exploiter des données de la littérature qui ont confirmé la validité et l'intérêt de cette méthode pour expliciter les 2 principaux phénomènes ayant lieu lors du dépôt d'une goutte sur une surface poreuse.

- Ces analyses et conclusions tendent à mettre en évidence un effet de colmatage des porosités fines des pâtes cimentaires plutôt qu'un effet hydrophobe $\mathrm{du}$ BA. D'autres analyses comme la bulle captive sont en cours pour confirmer ces conclusions. Les mesures de porosité à l'eau et au mercure ont été faites mais ne permettent pas d'observer d'importantes différences pour d'aussi faibles concentrations de BA. Les résultats des essais d'analyse de rugosité, porosité et comportement face à l'eau feront l'objet de futures publications. Finalement, les modifications de ces surfaces devront permettre une modification de leur bioréceptivité qui est étudiée par une équipe partenaire du projet SEPOLBE.

Remerciements. Ce travail est effectué dans le cadre de l'ANR (AAP CDII 2012 : projet SEPOLBE « Substances Extracellulaires Pour les BEtons $»)$. Les auteurs remercient chaleureusement l'ANR ainsi que les pôles de compétitivité 


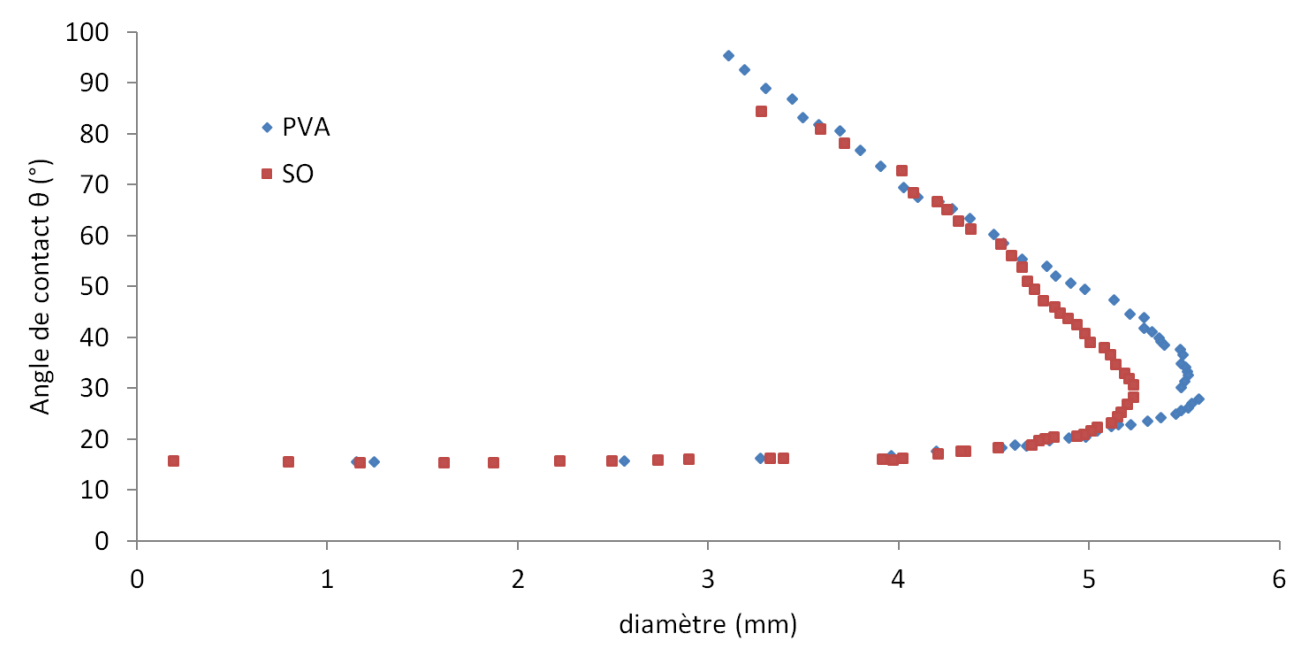

(a)

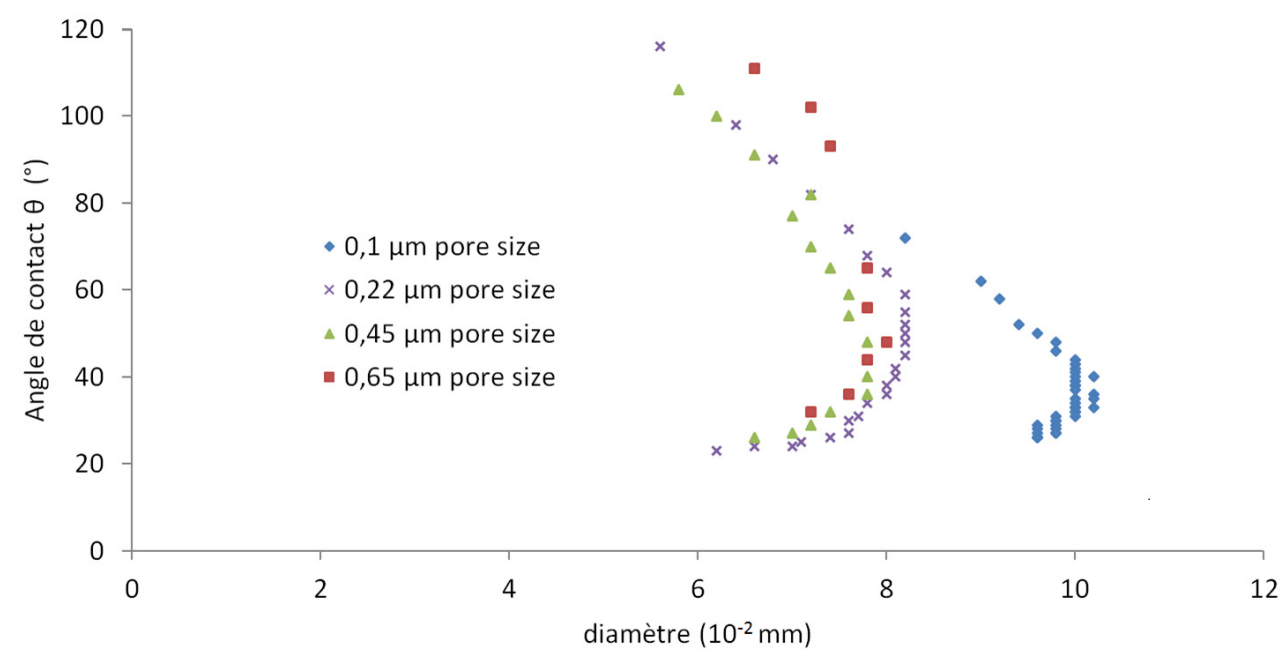

(b)

Fig. 15. (a) Goutte PVA et SO sur UDMAT [21], (b) Goutte de G/HG sur papier [23].

Fig. 15. (a) PVA et SO drop on UDMAT [21], (b) G/HG drop on paper [23].

ENERGIVIE, HYDREOS et ADVANCITY qui ont labellisé ce projet.

\section{Références}

[1] C. Gaylarde, M. Ribas Silva, T. Warscheid, Mater. Struct. 36 (2003) 342-352

[2] O. Guillitte, Sci. Total Environ. 167 (1995) 215-220

[3] F. Fritz-Feugeas, A. Cornet, B. Tribollet, Biodétérioration des matériaux? Action des microorganismes, de l'échelle nanométrique à l'échelle macroscopique, Ellipses Marketing, Paris, 2008

[4] A.G. Thu Hien Tran, Int. Biodeterior. Biodegrad. 79 (2013) 73-80

[5] M.A. Shirakawa, I.B. Beech, R. Tapper, M.A. Cincotto, W. Gambale, Int. Biodeterior. Biodegrad. 51 (2003) 8392

[6] M.Á.C.-T. Sandra Manso, Sci. Total Environ. 512-513 (2015) 444-453
[7] S. Roux, N. Bur, B. Tribollet, F. Feugeas, Matériaux Technique 99 (2011) 573-580

[8] S. Roux, B. Tribollet, N. Serres, A. Lecomte, F. Feugeas, Restor. Build. Monum. 19 (2013) 163-170

[9] H. He, N. Serres, T. Meylheuc, F. Feugeas, Matériaux Technique 101 (2013) 105

[10] P. Di Ciccio, A. Vergara, A.R. Festino, D. Paludi, E. Zanardi, S. Ghidini, A. Ianieri, Food Control 50 (2015) 930-936

[11] C. Yue, B. Yang, J. Bionic Eng. 11 (2014) 589-599

[12] S. Soleimani, O.B. Isgor, B. Ormeci, Cem. Concr. Res. 53 (2013) 229-238

[13] C.S. Butler, J.P. Boltz, 3.6 - Biofilm Processes and Control in Water and Wastewater Treatment, in Comprehensive Water Quality and Purification, S. Ahuja, (Ed.), Waltham, Elsevier, 2014, pp. 90-107

[14] F. Hamadi, H. Latrache, Colloids Surf. B Biointerfaces 65 (2008) 134-139 
[15] D.R. Absolom, F.V. Lamberti, Z. Policova, W. Zingg, C.J. van Oss, A.W. Neumann, Appl. Environ. Microbiol. 46 (1983) 90-97

[16] A.S.D. Al-Radha, D. Dymock, C. Younes, D. O'Sullivan, J. Dent. 40 (2012) 146-153

[17] C. Munzer, H. He, N. Serres, S. Roux, T. Meylheuc, A. Lecomte, F. Feugeas, Bioréceptivité des matériaux cimentaires - protocole de préparation et analyses des surfaces en fonction du temps, Rencontres Universitaires de l'AUGC, Orléans, 2014

[18] G. Wolansky, A. Marmur, Colloids Surf. Physicochem. Eng. Asp. 156 (1999) 381-388
[19] T.S. Meiron, A. Marmur, I.S. Saguy, J. Colloid Interface Sci. 274 (2004) 637-644

[20] A.J.B. Milne, A. Amirfazli, Adv. Colloid Interface Sci. $170(2012) 48-55$

[21] K.L. Apedo, C. Munzer, H. He, P. Montgomery, N. Serres, C. Fond, F. Feugeas, Mater. Charact. (2014)

[22] S.M. Kumar, A.P. Deshpande, Colloids Surf. Physicochem. Eng. Asp. 277 (2006) 157-163

[23] A. Clarke, T.D. Blake, K. Carruthers, A. Woodward, Langmuir 18 (2002) 2980-2984 